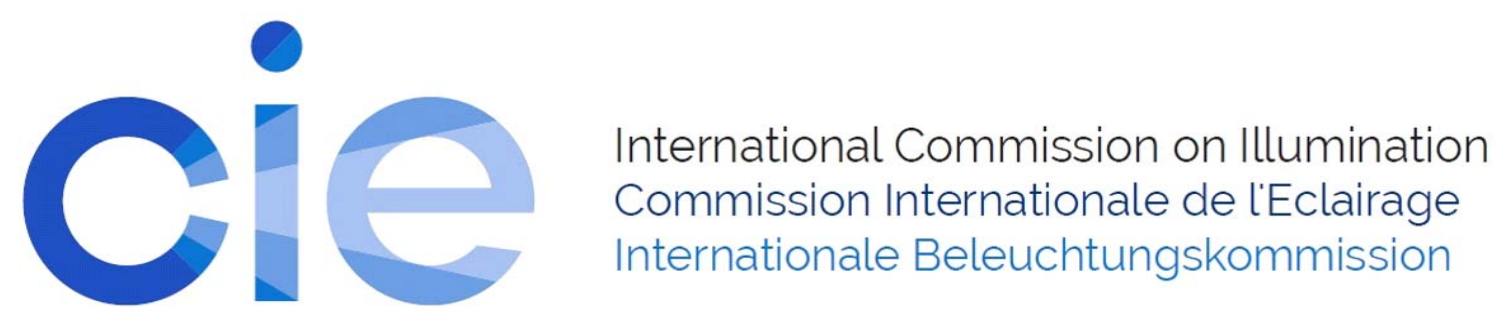

PP07

\title{
SHADE-FREE TEXTURE ACQUISITION FOR 3D SCANNING SYSTEM
}

\author{
Tzung-Han Lin et al.
}

DOI 10.25039/x46.2019.PP07

from

CIE x046:2019

\section{Proceedings}

of the

29th CIE SESSION

Washington D.C., USA, June 14 - 22, 2019

(DOI 10.25039/x46.2019)

The paper has been presented at the 29th CIE Session, Washington D.C., USA, June 14-22, 2019. It has not been peer-reviewed by CIE.

(C) CIE 2019

All rights reserved. Unless otherwise specified, no part of this publication may be reproduced or utilized in any form or by any means, electronic or mechanical, including photocopying and microfilm, without permission in writing from CIE Central Bureau at the address below. Any mention of organizations or products does not imply endorsement by the CIE.

This paper is made available open access for individual use. However, in all other cases all rights are reserved unless explicit permission is sought from and given by the CIE.

CIE Central Bureau

Babenbergerstrasse 9

A-1010 Vienna

Austria

Tel.: +4317143187

e-mail: ciecb@cie.co.at

www.cie.co.at 


\title{
SHADE-FREE TEXTURE ACQUISITION FOR 3D SCANNING SYSTEM
}

\author{
Lin, T.H., Chan, K.L., Chen, H.S. \\ National Taiwan University of Science and Technology, Taipei, CHINESE TAIPEI \\ thl@mail.ntust.edu.tw
}

DOI 10.25039/x46.2019.PP07

\begin{abstract}
Nowadays, 3D scanning technology is mature to obtain quality 3D shapes of real objects. However, to obtain a uniform and shade-free texture is still a challenging task, particularly for commercial products. We propose a practical solution in our 3D scanner to capture 3D object with highly uniform shade. This scanner utilizes a swinging laser in front of the cameras to scan 3D shape, and then captures additional colour images for texture. Those images are again adjusted according to the scanned 3D shape. Based on this design, the colour uniformity of scanned 3D object is significantly improved.
\end{abstract}

Keywords: Texture, Shade-free, 3D Scanning

\section{Background}

Our objective is to obtain uniform shades of the $3 \mathrm{D}$ object during the scanning process. Our proposed 3D scanner, which is a self-developed device, consists of two colour cameras, whose resolution are both $720 \times 1280$ pixels, one slit laser, and LEDs as external active illumination. For a commercial 3D scanner, it is unlikely to acquire the scattering or reflection distribution of object's surface, such as BRDF or BSDF models. The acquired surface texture is therefore not uniform. To obtain the native colour of a real object in a single shot, a practical solution based on shade compensation is proposed. This method simply removes the shade effect in every taken image under a known illumination condition.

\section{System configuration}

Our scanning system consists of several off the shelf components as shown in Figure 1. All components are controllable to obtain quality colour 3D objects. In the scanning pipeline, we have two independent processes. One is collecting 3D point clouds from eight directions. The other is taking colour images corresponding to all scanning directions.

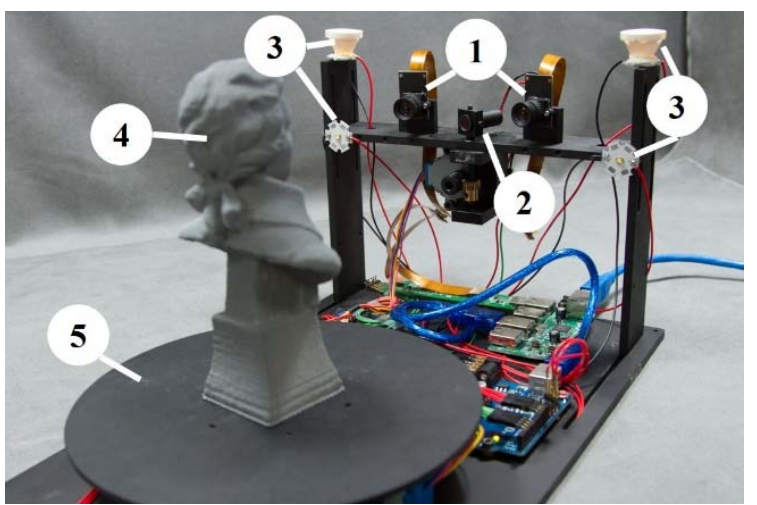

Figure 1 - Our scanning system consists of 1). Two cameras as a stereo set, 2). Sweeping laser driven by a step motor, 3). LEDs, 4). Scanned target and 5). Turntable.

\section{$2.13 \mathrm{D}$ scan process}

To determine 3D points, the stereo camera will take sequential synchronized stereo-pair images. And, the coordinates of $3 \mathrm{D}$ points are calculated based on epipolar geometry. In addition, all $3 \mathrm{D}$ points are converted into triangle meshes and stitched together. To form $3 \mathrm{D}$ range data, the 
swinging laser will vertically cast definite line features on 3D object's surface during scan process. And two synchronized cameras of the scanner will observe the vertical line features to determine 3D points in each frame. In other words, one range images data will be generated by scanning the target object from one direction. In our configuration, eight range data are enough to represent the shapes of most 3D objects.

In most 3D scanners, the cameras are monochromic in order to have better 3D performance. However, it sometimes suffers from either lack of colour textures or misalignment of additional colour cameras. For our system, to have a compromised quality, the colour cameras are used for both $3 \mathrm{D}$ and textures acquisition. Therefore, an additional colour image is then taken from one of the cameras in 3D scan process, and it has exactly the same coordinate with range data. Thus, a disparity-free texture image is collected. The turntable in the scanner will rotate the object to eight specific positions. There are totally eight range images are acquired to be integrated into a complete model by Poisson surface reconstruction (Lin, 2014).

\subsection{Colour calibration}

In colour calibration, we utilised X-Rite colour chart for reference. The colour chart is put on the centre of turntable and perpendicular to the viewing direction of one of camera as shown in Figure 2. Another white board is utilised to recorded the intensity of reflectance. Our colour calibration follows Hung's method (Hung, 1993) which is a second order polynomial regression for colour transformation matrix. In practice, the $X Y Z$ values of 24 checkers of the colour camera are converted from the raw image under D65 light. The transformation equation is written as equation (1) to convert the observed values [A] into reference values [B]. And the colour transformation matrix in equation (1) can be solved by least square method. Our polynomial form consists of 9 components as in equation (2). Therefore, the size of matrix [M] will be 9 by 3. Generally speaking, the refraction behaviour for lenses of cameras is not uniform, and it will induce non-uniform intensity distribution of image. To improve the uniformity of our colour image, a white board is used to recorded the intensity distribution. For every incoming image, the inversed mask corresponding to the intensity distribution is applied. As a result, uniform-shade and calibrated colour images are obtained.

$$
\begin{aligned}
& {[\mathbf{A}][\mathbf{M}]=[\mathbf{B}]} \\
& {[\mathbf{A}]=\left[X^{2}, Y^{2}, Z^{2}, X Y, Y Z, X Z, X, Y, Z\right]}
\end{aligned}
$$

where

[A] is the observed $X Y Z$ from colour camera;

$[\mathbf{M}]$ is the colour transformation matrix;

[B] is the reference $X Y Z$ of colour checker.
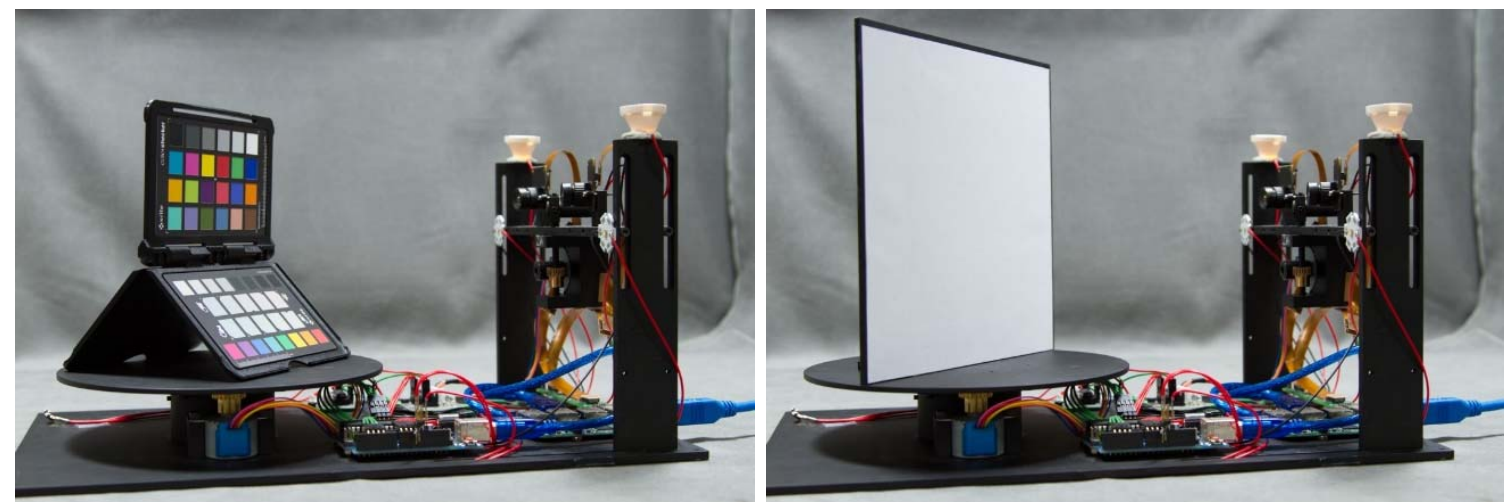

Figure 2 - Configuration of colour calibration of our proposed system

\subsection{Shade compensation}

For most 3D scanning systems, intrinsic factors are controllable to adapt extrinsic factors, such as environmental lighting, material properties and 3D geometry. The scan data of a real object usually suffer from uncertain colour appearance, particularly from shade and shadow. Those 
acquired texture images suffer from non-uniform illumination. After colour calibration, the influences to the shade of digital 3D objects are controlled. Another two factors affecting the shade of digital 3D objects are 3D geometry and reflection of materials of real objects. To avoid the influences due to these two factors, we use a sphere to recover the difference of intensity caused by the incident angle to object's surface. This method is basically a look-up table to compensate the reflection effect. An example shown in Figure 3 indicates that a 3D point $V$ with a normal vector $V_{n}$ vector will have an intensity compensation factor corresponding to the position $\mathrm{E}$ on the sphere.

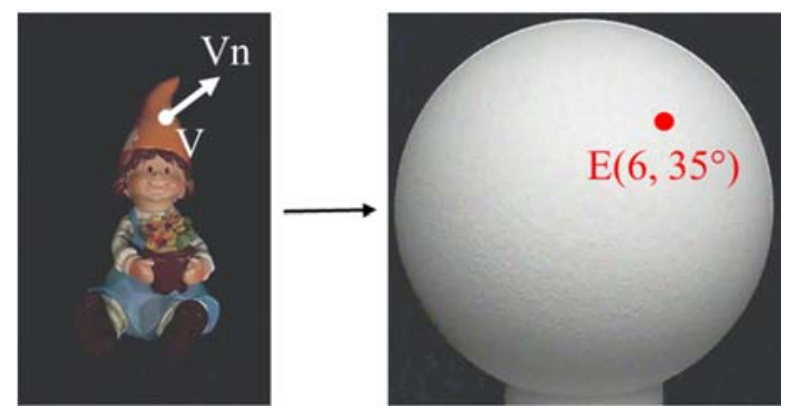

Figure 3 - A look-up table for recording the decayed intensities due to their normal vectors

\subsection{Shading in OpenGL}

To correct the non-uniform shade, we propose a compensation method based on openGL shading language to realistically simulate the light distribution. As mentioned in previous section, the look-up table, which records the decaying factors corresponding to surface normals, is generated. After previous calibration processes, we try to simulate the illumination distribution in the computer graphics environment. In our system configuration, two front LEDs are the direct lighting to 3D objects, and the other two LEDs are used as indirect lighting. Therefore, we set only two lights in openGL.

Since the scanner configuration is known, the positions of LEDs relative to the camera are known. Though their positions are roughly estimated, it does not affect result severely. In addition, we make a mathematic regression between the simulated image and the real image. Finally, the rendered image whose camera position is the same with corresponding image will be simulated. In practice, we utilize openGL shading language to carry out Phong shading method [3]. In other words, the simulated shade effect which is consistent to the real image is obtained. For every taken colour image, we adjust the lightness values by their corresponding simulated brightness. Finally, the shade effect in the real image will be removed by being simply divided by the simulated brightness.

For storing a digital 3D model, we directly use vertex colour render instead of a texture map from warping images in rending process. This is because the spatial resolution on the reconstructed object's surface, whose 3D positions are estimated by subpixel interpolation, is natively better than acquired images. To synthesize the surface colours and to suppress the illumination discrepancy among several scanning directions, a multiple-view blending method which follows a weighting function of surface normals is also carried out. As a result, the shade caused by the reflection of light to the surface of the test object is successfully supressed. And the uniformity of colour in synthesized 3D model is significantly improved.

\section{Result}

\subsection{Colour calibration}

In colour calibration results, the averaged colour difference between the original image and the reference colour chart is $\Delta \mathrm{E}^{*}{ }_{94}=33.3$. After calibration, the difference significantly drops to $\Delta \mathrm{E}^{*}{ }_{94}=2.7$, which is acceptable for most applications.

\subsection{Simulated shade images}

To proof the consistence between the simulated image and the real image, we compared them by using a white board in front of one of cameras. Since our cameras and LEDs on the rig are 
symmetric, the LEDs relative to one of cameras will be asymmetric. This is why the brightness distribution on the real image is asymmetric as in Figure 4(a). The brightness values in the distribution map are intentionally normalized for convenience. From the pair comparison of brightness distributions, the simulated brightness is consistent with the real. The difference between them is regarding the glossiness of materials. In Phong's mathematic method, the glossiness is controlled by one exponential factor of a cosine function. This exponential factor differs in various materials. And it can be readily estimated by taking reference images.

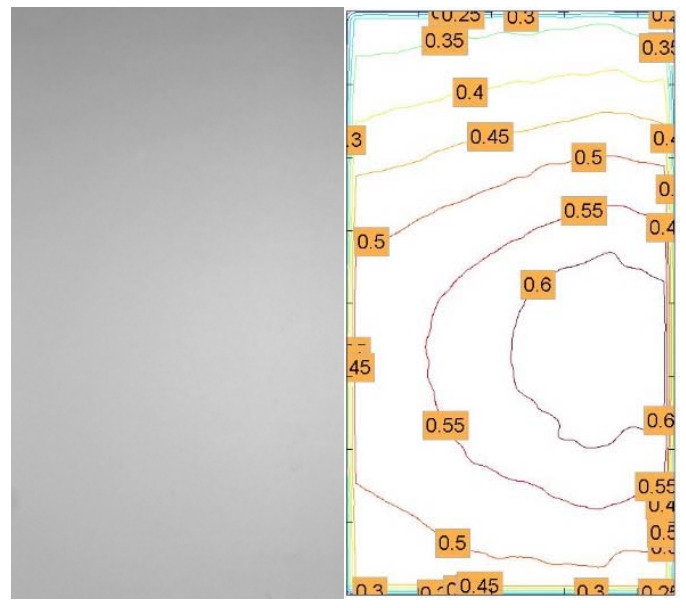

(a) real image and brightness distribution

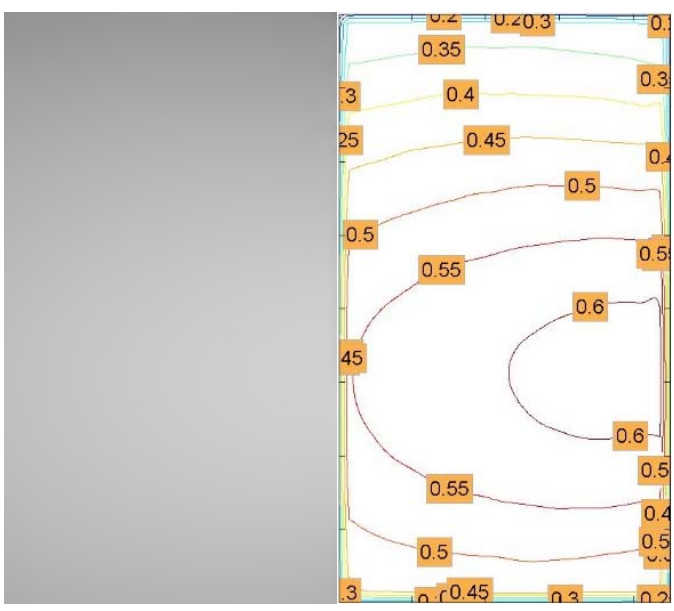

(b) simulated image and brightness distribution

Figure 4 - The brightness comparison between the real and simulated images

\subsection{Shade-free images}

In the result of generating shade-free images, one example in Figure 5 (c) comes from the combination of the original image and rendered image. The shade effect in the original image, as Figure (a), can be observed on the margin on silhouette. Since the surface normal has large incline angle to the viewing direction, the brightness drops severely. This effect is also found in the simulated image as Figure (b). The phenomenon induces that the gain for pixel around the silhouette margin has to be greater. However, in the critical case on margin, the gain value will cause over-saturation in the synthesized image. In those cases, we clap a maximum value of data range and add one attenuation function to obtain a smooth and reasonable drop of shade. Thought, the over-saturation case happens, it is still acceptable. The is because the texture colours in 3D models are blended from eight images according to a weighting function of viewing directions.

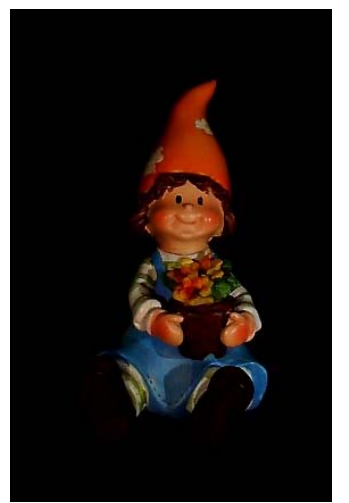

(a)

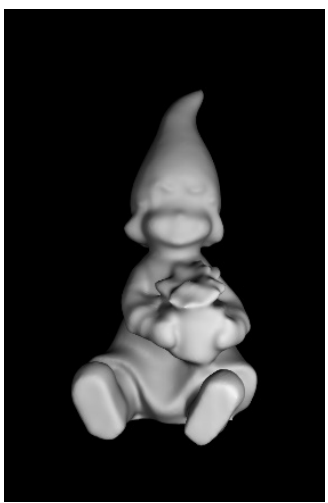

(b)

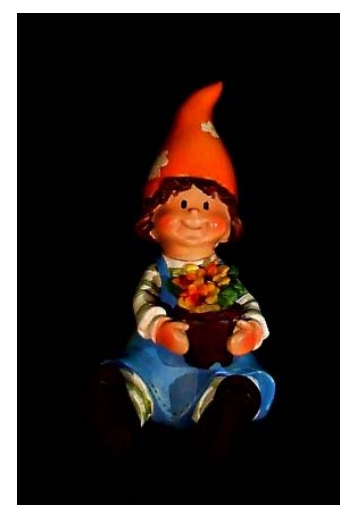

(c)

Figure 5 - The shade-free image of (c) is synthesized from the original image of (a) and the render intensity image of (b). 


\subsection{Shade-free 3D models}

In synthesized a shade free 3D model, we initially render the model with monochromic shade under the same parameters corresponding to the real camera. The extrinsic parameter of the virtual camera is readily to be the same as the real camera. However, the intrinsic parameter including lens distortion can not be directly implemented in openGL. We applied an un-distortion operation to the original images as well as its shade-free images. Then, eight images are cast on the 3D model according to a weighting blending function. As a result, the consistence between simulated and captured images is controlled as accurate as sub-pixel level. Figure 6 (a) shows the scanning result of our system. The object is made of earthenware with colour coated surface. Figure 6 (b) is the result of a textured model with conventional shade images. It can be found that the uniformity of colour is slightly improved due to the weighting blending function in synthesising textures. Figure 6 (c) presents the synthesised result of proposed method. With comparison to previous method, the rendering result shows a smooth and uniform appearance.

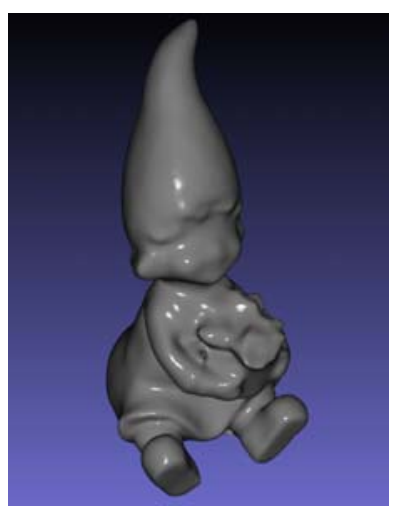

(a)

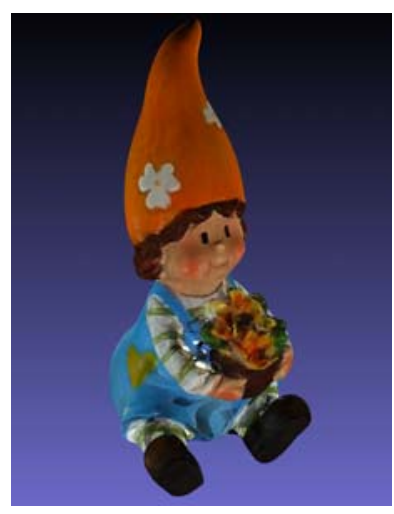

(b)

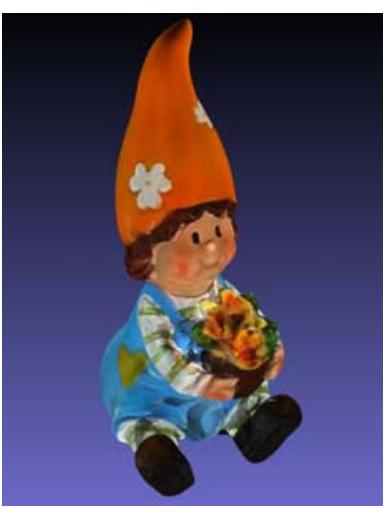

(c)

Figure 6 - 3D model from our scanning system. (a) 3D model without texture, (b) 3D model with blended shade texture, and (c) 3D model with blended shade-free texture.

\section{Conclusion}

We successfully carried out a 3D scanning system which is capable to acquire shade-free images based on three calibration processes. With regard of the limitation, our method is so far not able to deal with self-occluded shadows and glossy surfaces. Nevertheless, it is very likely to be overcame in the future by ray-tracing and high dynamic range image.

\section{Acknowledgments}

This work was supported in part by the Ministry of Science and Technology of Taiwan under Grant No. MOST 107-2221-E-011-137-.

\section{References}

Hung, P. C. 1993. Colorimetric calibration in electronic imaging devices using a look-up-table model and interpolations. Journal of Electronic Imaging, 2(1), 53-61.

Lin, T. H. 2013. Resolution adjustable 3D scanner based on stereo cameras. Asia Pacific Signal and Information Processing 2013.

Phong, B. T. 1975. Illumination for computer generated pictures. in Communications of the ACM, 18(6), 311-317. 